\title{
II. ENTRE DEUX CARDINAUX: LES INSTRUCTIONS PRINCIPALES DONNÉES AUX PLÉNIPOTENTIAIRES FRANÇAIS EN 1643
}

D’une manière générale, les instructions données aux ambassadeurs français constituent une source primordiale pour connaître les notions acquises sur l'Allemagne à la cour de Francel. Cela vaut en particulier pour les instructions données aux plénipotentiaires français en Westphalie, qui sont le résultat d'une réflexion approfondie sur les objectifs et les méthodes de la politique française au congrès de la paix; des premières ébauches à la rédaction définitive de ce chef d'œuvre de la diplomatie française, environ sept ans se sont passés. Commencée en 1636, la rédaction fut terminée en 1643; Richelieu en était le principal auteur, mais il incombait à Mazarin d'y mettre la touche finale?

Deux historiens allemands, Fritz Dickmann et Kriemhild Goronzy, ont reconstitué avec un souci de précision admirable l'histoire complexe de ce document extraordinaire $^{3}$. Ils concluent que bien que l'original de l'instruction principale pour le congrès de Westphalie soit daté du 30 septembre 1643, pendant le ministère du cardinal Mazarin, ce texte est en réalité presque entièrement l'œuvre de Richelieu, sorte de »testament de politique étrangère « du cardinal ${ }^{4}$. En fait, l'organisation d'un congrès de la paix générale était prévisible depuis 1634 , avant même que la France ne déclarât la guerre à l'Espagne, et certaine depuis 1641, de sorte que Richelieu était obligé de préparer minutieusement cet événement crucial pour l'avenir du royaume. Au moment de son décès, le 4 décembre 1642, il légua à Mazarin un grand nombre de projets d'instructions presque entièrement achevées et traçant les lignes principales de la future politique étrangère française. Dickmann et Goronzy estiment que la paix de Westphalie serait ainsi devenue »la

1 Garden dresse ce bilan en se référant aux seules instructions du temps de Louis XIV, mais il vaut également pour la première moitié du XVIIe siècle et tout le XVIIIe, cf. Guillaume, comte DE GARDEN, Histoire générale des traités de paix et autres transactions principales entre toutes les puissances de l'Europe depuis la paix de Westphalie [...], 15 vol., Paris [18481887], t. I, p. XXXII, n. 1: les instructions de Louis XIV »résument toutes les notions acquises sur les différentes puissances par les missions antérieures, et [...] renferment un ensemble de desseins, de vues et de motifs raisonnés, propres à faire connaître l'esprit et les tendances du cabinet de Versailles

2 Ces instructions ainsi que les projets et les différentes additions ont été publiés: Fritz DickMANN et Kriemhild GoRONZY (éd.), Die französischen Instruktionen (1636-1643), dans: APW I 1, p. 1-189, ici document no 5 (Die Ausfertigung der Hauptinstruktion für Münster, Paris 1643 September 30), p. 58-123.

3 Ils résument les résultats de leurs études dans l'introduction à l'édition du document (ibid., p. 1-16).

4 »Die französische Hauptinstruktion für den Westfälischen Frieden trägt das Ausfertigungsdatum vom 30. September 1643, stammt also aus der Amtszeit Mazarins. In Wirklichkeit ist sie aber ausschließlich ein Werk Richelieus - wenn man so will: sein außenpolitisches Vermächtnis« (ibid., p. 1). 
fin brillante « de la politique étrangère de Richelieu 5 . Si cette conclusion nous paraît incontestable à l'égard des instructions de 1643, le chemin qui devait mener à la paix fut encore long. Ainsi, nous la mettrions au conditionnel quand il s'agit d'interpréter les résultats obtenus en 1648: en effet, la France renonça, au cours des négociations, au moins à l'un des objectifs majeurs des conceptions de la paix de Richelieu concernant la sûreté du traité, à savoir les projets des ligues allemande et italienne ${ }^{6}$.

En revanche, en ce qui concerne l'instruction principale, Mazarin ne retrancha que peu de passages du dernier projet de Richelieu et en ajouta d'autres moins importants; le secrétaire d'État aux Affaires étrangères, Henri-Auguste de Loménie, comte de Brienne, rédigea le préambule. En 1641, Richelieu avait repris les instructions minutées pour le congrès de Cologne en 1636 et 1637 . Il y a donc une filiation des différents projets permettant de saisir les continuités et les modifications des conceptions de la paix depuis le tout début de la guerre contre l'Espagne et l'empereur. En comparant ces différentes étapes, on constate que les continuités sont beaucoup plus importantes que les changements; par ailleurs, les éditeurs des documents concernés ont pu établir le fait que, pour des raisons paléographiques et substantielles, les projets minutés du vivant du cardinal sont essentiellement l'œuvre personnelle de Richelieu, non de ses conseillers?.

Pour être précis, il faut noter que Richelieu avait rédigé, en 1641 et 1642, au moins trois projets d'instructions pour Mazarin et d'Avaux ${ }^{8}$, initialement prévus pour représenter la France au congrès de la paix, ainsi qu'un supplément d'instructions qui envisageait des solutions alternatives à certains problèmes de conférences de la paix ${ }^{9}$ et un mémoire concernant les droits de la couronne de France sur différents territoires en Europe $^{10}$ (ces pièces datent également de 1641-1642); à cela s'ajoutaient les trois projets d'instructions écrits en 1636 et 163711 . En ce qui concerne les différents projets, les éditeurs distinguent deux étapes qu'ils attribuent au ministériat de Richelieu, tandis que la troisième et dernière étape ( $\mathrm{y}$ compris les deux originaux de 1643 qui furent peut-être donnés à d'Avaux et à Servien) fait partie du ministère de Mazarin. Les trois copies des projets du premier groupe (congrès de Cologne) ont été rédigées entre décembre 1636 et mars 1637; les éditeurs concluent d'un ouvrage de Pierre Dupuy publié en 1655 que Ri-

5 »Die weitausgreifenden Pläne und Gedanken dieses Staatsmannes in dem fast vollendet hinterlassenen Instruktionsentwurf wurden für seinen Nachfolger im Amt richtungweisend; Richelieu hatte für alles, was Frankreich im Westfälischen Frieden erstrebte und meist auch erreichte, die Grundlinien vorgezeichnet: die Pax Westphalica wurde damit zum glanzvollen Abschluß seiner Außenpolitik« (ibid.).

6 Ce point mérite d'être soulevé à nouveau à la fin de cette partie du livre.

7 DickManN, GoRonzy (éd.), Die französischen Instruktionen, p. 2-3 et p. 14.

8 Certaines parties qui n'ont pas été insérées dans la version finale de l'instruction sont publiées ibid., documents nos 6 à 9 , p. 123-139.

9 Publié ibid., document no 11 , p. 150-158.

10 Publié ibid., document $n^{\circ} 12$, p. 159-189.

11 L'une des copies est publiée ibid., document $n^{\circ} 3$, p. 38-55; une note sur ce projet se trouve ibid., document $n^{\circ} 4$, p. 56-58. 
chelieu se fonda en partie sur des mémoires écrits en février et mars 1637 par Théodore Godefroy, qui fut par la suite conseiller juridique des ambassadeurs français en Westphalie. Le premier projet du deuxième groupe daterait d'août ou de septembre 1641; pendant les mois suivants, il fut entièrement corrigé. Les deux projets suivants du même groupe, beaucoup plus volumineux que les précédents, ont encore été rédigés sous Richelieu, probablement à partir de décembre 1641. La rédaction du deuxième projet fut terminée à la mi-juillet 1642; les parties les plus importantes dateraient de la fin de 1641 et du tout début de 1642 (rappelons que d'après les préliminaires de Hambourg, le congrès devait commencer le 25 mars 1642$)^{12}$. La collaboration de Mazarin à la rédaction des projets fut antérieure à la mort de Richelieu. En effet, ce dernier avait chargé son successeur présomptif de mettre au propre le dernier projet d'instructions, en octobre 1642. À la fin de l'été 1643, Mazarin mit la dernière main aux projets d'instructions, sans les remanier profondément ${ }^{13}$; le texte définitif date, comme nous l'avons déjà dit, du 30 septembre $1643^{14}$. Parmi les conseillers qui ont collaboré à ses projets en fournissant au cardinal de Richelieu les mémoires et les informations historiques et juridiques nécessaires, on note encore le nom de Théodore Godefroy. Le 20 mars 1642, le secrétaire d'État aux Affaires étrangères, Chavigny, envoya une lettre de Narbonne à Godefroy accusant réception de deux mémoires; c'était apparemment dans le cadre de la rédaction des instructions qu'il lui en demanda d'autres ${ }^{15}$. De toute évidence, Godefroy était pressenti comme conseiller des plénipotentiaires français ou, du moins, devait préparer leur mission. Le 6 septembre 1641, Chavigny lui adressa une autre lettre, dans laquelle il ordonna à l'historiographe du roi de se procurer les documents nécessaires devant servir aux ambassadeurs français susceptibles de partir sous peu à Münster ${ }^{16}$.

Afin d'impliquer l'autorité et la responsabilité personnelles du roi, Richelieu décida de donner aux ambassadeurs français les copies de deux lettres signées par Louis XIII, dans lesquelles le roi justifiait la guerre contre l'Espagne (première lettre, envoyée de Chantilly à Richelieu le 4 août 1634) et fixait les principales conditions de la paix (deuxième lettre, expédiée le 2 janvier 1642) ${ }^{17}$. Mazarin exécuta très certainement ce dessein ${ }^{18}$.

12 Pour l'histoire des différents projets et pour les conclusions des éditeurs, cf. ibid., p. 5-14 et p. 24-37.

13 Cf. ibid., p. 14.

14 Publié ibid., document no 5, p. 58-123. Cette instruction pour Münster est complétée par une instruction particulière relative aux négociations de La Haye avec les Provinces-Unies (les ambassadeurs français devaient passer par là avant d'arriver en Westphalie), publiée ibid., document $n^{\circ} 10$, p. 139-149.

15 BNF, F.fr., Nouvelles acquisitions (nouv. acq.) 5163 fol. 9; cité dans APW I 1, p. 11, n. 3 .

${ }^{16}$ Institut de France (IF), Collection Godefroy (Coll. Godefroy) 34 fol. 200; cité dans APW I 1, p. 9.

17 Publiées ibid., documents $n^{\circ s} 1$ et 2 , p. 17-23. Au sujet de la première lettre, cf. aussi le chapitre précédent.

18 Cf. ibid., p. 3, n. 1, p. 17 et p. 21. 
L'acte de Louis XIII fixant les principales concessions que la France était prête à faire au congrès de la paix, daté de 1642, fut demandé par Richelieu, qui soumit des questions et des problèmes précis à la décision du souverain; le roi ajouta ses résolutions et ses remarques en marge du texte et le signa. Concrètement, Richelieu nota les demandes qu'ont attendait de la part des Impériaux et des Espagnols; en les approuvant ou en les refusant et en posant des conditions aux éventuelles concessions françaises, le roi détermina la politique que Mazarin, alors prévu comme ambassadeur plénipotentiaire français, devait mener au congrès de la paix. Mais en choisissant les demandes qui étaient soumises à la décision du roi, Richelieu pouvait suggérer les sujets qui lui tenaient à cœur. Ces revendications potentielles des ennemis de la France concernaient deux aspects différents: premièrement, la restitution de certains territoires occupés par la France à leurs propriétaires antérieurs; deuxièmement, les relations entre la France et ses alliés après la conclusion de la paix. Les deux aspects avaient un rapport direct avec le problème de la sûreté du traité, dans la mesure où ils faisaient partie de la politique des passages et des protections conçue par le cardinal ou de ses projets de sécurité collective. Ce document reflète donc non seulement les positions du roi, mais aussi les préoccupations et les conceptions de la paix du cardinal ${ }^{19}$. Deux demandes concernaient la politique allemande de la France. La première avait pour objet la restitution de Brisach et des autres places fortes occupées en Alsace et en Allemagne aux Impériaux. Certes, ce problème était d'une importance majeure pour la défense du royaume. Mais, dans ce cas-là, la réponse du roi paraît encore plus intéressante que la demande de Richelieu, puisqu'elle révèle le caractère injustifié de l'hypothèse historiographique selon laquelle le cardinal aurait imaginé et mené une politique délibérément expansive afin de faire du Rhin la frontière du royaume. En effet, Louis XIII décida: »Cela dépendra des avantages que l'on fera dans la Paix au Roy, ou ceux qu'il aura, quand on fera la Paix « ${ }^{20}$. Pour la France, la rétention de l'Alsace et de certaines forteresses allemandes n'était donc pas une conditio sine qua non de la conclusion du traité de paix. Il n'en va pas de même pour les alliances avec les princes du Saint-Empire que le roi n'était pas prêt à abandonner.

À la question de Richelieu concernant la demande des Impériaux et des Espagnols »qu'à l'avenir le Roy renonce à l'alliance des Suédois et de tous autres Princes d'Alemagne qui ont fait la guerre avec luy«, Louis XIII répondit: »Une telle proposition estant contre l'honneur de Sa Majesté, il n'y a pas d'apparence d'y entendre, et c'est chose honteuse de la proposer « ${ }^{21}$. Comme la France avait

${ }^{19}$ Le dernier paragraphe de la partie du texte qui était signée par Louis XIII l'avertissait de l'importance de réfléchir, avant de prendre ses décisions, sur le problème de la sûreté du traité: »Il plaira au Roy faire sçavoir ses pensées sur ces sujects et avoir esgard en ses résolutions à l'avenir comme au présent, considérant bien quelles seuretéz il veut demander pour se garentir de la coustume qu'ont les Espagnolz de n'observer point les Traitéz qu'ilz font, mais de les rompre aussytost qu'ilz le peuvent faire avec avantage « (APW I 1, no 2, p. 23).

20 Ibid., p. 22.

21 Ibid., p. 23. 
légitimé sa déclaration de guerre avec la protection des libertés germaniques, il n'était pas possible de renoncer à l'alliance avec les états de l'Empire sans discréditer à la fois cette justification de l'intervention militaire en Allemagne et la sécurité de la France après la signature de la paix. En revanche, ce document ne faisait point allusion aux droits de l'empereur. En effet, Richelieu voulait limiter l'autorité de l'empereur en renforçant les droits des ordres, mais son objectif n'était pas de l'affaiblir au point que les protestants obtiennent l'hégémonie dans l'Empire 22 .

Si l'on peut dire que Richelieu considérait l'Espagne comme l'ennemi principal du royaume de France, cela est vrai aussi pour Mazarin et pour la politique française à Münster; Konrad Repgen remarque à juste titre: »Pour la France, l'objet principal au congrès de la paix de Westphalie n'était pas l'empereur, ni l'empereur et l'Empire, mais: l'Espagne « ${ }^{23}$. Pour cette raison, les instructions principales données aux ambassadeurs de France le 30 septembre 1643 mettent l'accent sur une éventuelle rupture du futur traité de paix par l'Espagne et proposent divers moyens pour rendre la paix plus sûre. En effet, la quatrième section - la première qui concerne concrètement les négociations au congrès de paix - met le problème de la sécurité au premier plan des différends à régler: »La première chose qui doit estre mise sur le tapis en la négociation de la Paix est d'arrester les seuretés du Traitté qui doit estre fait « ${ }^{24}$. Après la mort du cardinal de Richelieu survenue en décembre 1642 et celle de Louis XIII en mai 1643, les orientations de la politique étrangère française ne changèrent donc pas radicalement du jour au lendemain. La politique de la régence, dirigée par Anne d'Autriche et le cardinal Mazarin, reprit les projets d'instructions que Richelieu avait esquissés. Aussi les projets de sécurité collective conçus par Richelieu furent-ils inscrits à l'ordre du jour du congrès, bien que Mazarin ne les soutînt pas avec la même ferveur que son prédécesseur l'eût peut-être fait ${ }^{25}$.

22 Cf. TISCHER, Französische Diplomatie, p. 184-185.

23 „Das politische Hauptthema Frankreichs im Westfälischen FriedenskongreB hieB nicht: Kaiser, oder: Kaiser und Reich, sondern: Spanien «, Konrad REPGEN, Vorwort, dans: BRAUN, APW II B 5/1, p. VII-VIII, ici p. VII. Cependant, Hermann Weber note que l'empereur - au lieu du roi d'Espagne - fut le »premier partenaire de la France et de ses alliés« (»der erste Partner Frankreichs und seiner Verbündeten «) au congrès de la paix, que ce fut avec lui seul qu'elle conclut la paix et que ce changement durable de paradigme, qui devait caractériser l'avenir, fut la principale conséquence de la politique allemande de Richelieu, WEBER, Richelieu und das Reich, p. 45.

24 APW I 1, no 5, p. 70-71.

25 Certes, les termes de »sécurité collective«, voire de »système de sécurité collective«, sont anachroniques. Externbrink les a rejetées pour cette raison, critiquant les travaux de Weber et de Dickmann sur la politique du cardinal, cf. ExTERnBRINK, Le cơur du monde. Néanmoins, Weber, qui est probablement le meilleur connaisseur de Richelieu parmi les historiens allemands de l'après-guerre, et Dickmann, le premier à avoir appliqué ces notions à la politique de Richelieu - le concept de »Kollektive Sicherheit« est aussi utilisé dans son édition des instructions du 30 septembre 1643 (APW I 1, document no 5, ici p. 70) -, ont pu démontrer de manière convaincante que, sans connaître le mot, Richelieu a bien inventé un système de sécurité collective. Le dernier en date qui sanctionne leur position est BABEL; cf. ID., 
Or, ce système de sécurité collective que la paix de Westphalie aurait dû instaurer - si la France avait pu imposer ses projets - était censé se fonder sur deux ligues: l'une devait comprendre les princes d'Italie, l'autre ceux d'Allemagne ${ }^{26}$. Dans la pensée de Richelieu, l'Allemagne jouait donc un rôle primordial dans la mesure où elle garantirait la France d'éventuelles contraventions espagnoles au traité de paix. L'Allemagne, c'était pour Richelieu les princes et les états de l'Empire; aussi longtemps que l'empereur appartiendrait à la maison d'Autriche, son seul appui dans l'Empire serait les ordres, surtout (mais pas exclusivement) les protestants. C'est la raison pour laquelle la diplomatie française devait veiller à ce que, par ce traité de paix, les princes allemands obtinssent le droit d'entretenir une armée et de conclure des alliances; en effet, la ligue d'Allemagne même eût été impossible sans qu'on accordât le ius foederis aux ordres. Pour protéger la France contre l'Espagne, le cardinal devait donc soutenir les ambitions constitutionnelles des ordres du Saint-Empire.

Selon sa conception, tous les princes, seigneurs, villes libres et républiques d'Italie et d'Allemagne devaient garantir la paix universelle, par la force des armes s'il en était besoin ${ }^{27}$. Ce passage de son instruction de 1641 (qui s'était déjà trouvé in nuce dans celle de la fin de 1636) fut textuellement repris dans la quatrième section de l'instruction que Mazarin rédigea en 1643 pour les négociateurs de Westphalie ${ }^{28}$. Même si ces instructions ne précisent pas que la France devait entrer dans les ligues proposées, il est évident qu'elles - la France et les ligues devaient former un »ensemble« capable d'affronter l'Espagne si les Espagnols voulaient perturber la paix ${ }^{29}$. Pourtant, Richelieu distinguait clairement les affaires italiennes et allemandes et proposait donc deux ligues différentes et indépendantes ${ }^{30}$; il est évident qu'une garantie universelle eût diminué les chances de réussite de ses projets. Tout en ayant pour but de conclure deux ligues, l'une en Italie et l'autre en Allemagne, l'instruction donnée aux ambassadeurs plénipotentiaires de France en 1643 ne précisait pas les éventuelles restrictions que l'on pouvait accorder aux alliés et aux ennemis ni la méthode précise à employer pendant la négociation. Ce passage correspond littéralement aux projets rédigés par Riche-

Deutschland und Frankreich im Zeichen der habsburgischen Universalmonarchie, p. 96. Pour la conception de la sécurité collective dans la pensée politique de Richelieu, cf. également HARTMANN, Rêveurs de paix?, p. 102-104; Klaus MALETTKE, Richelieus Außenpolitik und sein Konzept kollektiver Sicherheit, dans: Peter KRÜGER (dir.), Kontinuität und Wandel in der Staatenordnung der Neuzeit, Marbourg 1991 (Marburger Studien zur Neueren Geschichte, 1), p. 47-68; ID., Frankreich, Deutschland und Europa im 17. und 18. Jahrhundert, p. 275-285; ID., Konzeptionen kollektiver Sicherheit in Europa bei Sully und Richelieu, dans: August BUCK (dir.), Der Europa-Gedanke, Tübingen 1992 (Deutsch-italienische Studien, 7), p. 83-106, en particulier p. 95-106; ID., Die Entwicklung eines Systems.

$26 \mathrm{Cf}$. la quatrième section des instructions données aux ambassadeurs de France le 30 septembre 1643, APW I 1, document $n^{\circ} 5$, ici p. 70-72.

27 Cf. ibid., p. 71.

28 WeBER, Chrétienté et équilibre européen, p. 12.

29 Telle est l'interprétation de WeBER, ibid., p. 13.

30 Cf. APW I 1, document $n^{\circ}$ 5, p. 71-72. 
lieu, qui n'en avait jamais fixé les conditions exactes, puisqu'il savait très certainement que les pourparlers demandaient une certaine flexibilité.

Concrètement, la seule obligation des plénipotentiaires du roi consistait à concerter les conditions précises des ligues avec ses alliés. Les projets n'étaient qu'ébauchés, et, finalement, Richelieu ne pouvait plus veiller à leur exécution. Il n'en reste pas moins qu'ils constituaient un élément fondamental de la pax universalis, mais en même temps pax gallica et donc dans une certaine mesure antiespagnole ${ }^{31}$ conçue par l'Homme Rouge. Ce sont les mêmes instructions minutées par Richelieu lui-même et données sans aucune modification aux ambassadeurs de France en 1643 (en ce qui concerne ce passage) qui précisaient leur importance fondamentale pour la sûreté de la paix ${ }^{32}$.

Après les projets des ligues italienne et allemande, Richelieu et Mazarin voulaient imposer la libération de l'archevêque de Trèves avant de commencer les délibérations sur les autres différends; rappelons que pour libérer ce prince, la France était entrée en guerre. Or, il était non seulement nécessaire d'obliger les ennemis à rétablir ce prince électeur dans sa dignité et à lui rendre ses possessions afin de prouver la légitimité de la politique française depuis le début de la guerre, mais le roi pouvait aussi compter sur le soutien de Sötern dans la suite des négociations, après sa réintégration dans le collège des électeurs, et d'une manière générale dans les circonstances futures où il en aurait besoin. L'instruction de 1643, qui correspondait littéralement aux derniers projets d'instructions rédigés par Richelieu, en ce qui concerne l'électeur de Trèves, ne cachait pas cet espoir aux ambassadeurs de France. La légitimation juridique de l'intervention française en faveur de Sötern, que les ambassadeurs français étaient censés alléguer en négociant avec les Impériaux en 1643, correspondait très exactement à sa justification et à celle de son acceptation par l'électeur en 1635. En effet, selon l'argumentation de Richelieu (littéralement reprise par Mazarin), le roi de France avait pris, sur le plan constitutionnel, le rôle traditionnel de l'empereur, celui de protecteur d'un prince de l'Empire, dont le chef n'avait pu assumer cette fonction - protéger non seulement l'électeur, mais aussi la religion catholique - au moment de la conclusion du traité de protection entre Louis XIII et Sötern ${ }^{33}$. L'image de la situation constitutionnelle du Saint-Empire transmise par cette légitimation, celle d'un empereur faible, incapable d'assurer la sûreté des princes de l'Empire et de la religion catholique, dont il aurait dû être le premier défenseur comme »avocat de l'Église « (advocatus Ecclesiae), reflétait probablement la pensée du cardinal de Richelieu en 1635. Mais il savait très certainement que cette situation pouvait rapidement changer en faveur de l'empereur, donc de l'Espagne; les rapports de force entre l'empereur et les ordres n'étaient pas définitivement réglés, et il im-

${ }^{31}$ Certes, l'idée de paix supposait que l'Espagne devait elle aussi trouver sa place dans une chrétienté pacifiée. Mais pour cela il fallait qu'elle renonce à ses prétendues impulsions belliqueuses, de sorte que certaines clauses du traité devaient lui rendre impossible une politique offensive, voire aggressive, au détriment de la France et de ses alliés.

32 APW I 1, document $n^{\circ} 5$, ici p. 72.

33 Cf. ibid., p. 72-73. 
portait au roi de soutenir ces derniers et que ceux-ci contribuent à leur tour à sa sécurité en constituant une opposition interne au pouvoir impérial. Il est évident que Richelieu et Mazarin escomptaient conserver une clientèle française parmi les électeurs, les princes et les états de l'Empire après la signature de la paix. L'amitié d'un électeur, en l'occurrence l'archevêque de Trèves, était cruciale pour assurer à la France une influence certaine en Allemagne, au-delà de la guerre. Cependant, Richelieu avait déjà retranché de ses derniers projets d'instructions un passage soulignant les éventuelles conséquences du rétablissement de l'électeur sur les élections impériales.

En ce qui concerne les autres différends, on doit retenir l'ordre des matières proposé dans les instructions du 30 septembre 1643: le fait que l'Allemagne n'ait pas été au cœur des préoccupations françaises (bien que la postérité ait retenu le contraire) se manifeste, en effet, dans l'ordre donné aux ambassadeurs français de traiter d'abord des affaires d'Italie ${ }^{34}$. Sans aucun doute, les deux cardinaux - Richelieu aussi bien que Mazarin - considéraient l'Italie comme le »cœur du monde « ${ }^{35}$, puisqu'ils jugeaient que c'était en Italie que les Espagnols possédaient la plus belle partie de leur empire. L'Allemagne se trouvait ainsi reléguée au rang de théâtre »secondaire«; même si son importance pour la politique française avait très certainement augmenté depuis les années 1620 , la structure de cette conception des relations internationales qui faisait de l'Espagne le pivot de toutes les considérations ultérieures se reflétait donc toujours dans les instructions de $1643^{36}$.

Les autres questions allemandes - c'est-à-dire tous les différends concernant l'Allemagne en dehors du projet de la ligue allemande et de la restitution de l'électeur de Trèves - étaient abordées dans les sections XV à XVIII de ce document (il en comptait vingt-trois au total ainsi qu'une instruction particulière pour Mantoue et Savoie). Il convient d'étudier très attentivement ces instructions, puisqu'elles constituaient le legs de la politique allemande du cardinal de Richelieu, car les modifications dues à Mazarin dans ces quatre sections ont un caractère plutôt stylistique que substantiel ${ }^{37}$. La section XV ne se limitait pas à l'Allemagne, mais abordait également le problème du dédommagement de la couronne de Suède et son alliance avec le roi de France; ces problèmes étaient même présentés

${ }^{34}$ Cf. ibid., p. 73, début de la sixième section.

35 Pour la politique italienne de Richelieu, cf. ExTERnBRINK, Le cour du monde; pour la politique italienne de Mazarin durant le congrès de Westphalie et pour l'importance que ce cardinal originaire d'Italie continua d'attacher à son pays d'origine, cf., entre autres, Guido Braun, Päpstliche Friedensvermittlung am Beispiel von Piombino und Porto Longone, dans: Quellen und Forschungen aus italienischen Archiven und Bibliotheken 83 (2003), p. 141-206.

36 Pour la première période de la politique étrangère de Richelieu, avant la guerre de la Succession de Mantoue, Weber a parlé du Saint-Empire »comme [d']un théâtre secondaire et dépendant [de la politique espagnole] « (»zeigt sich das Reich zunächst als ein sekundärer, abhängiger Schauplatz der Richelieuschen Politik «, WEBER, Richelieu und das Reich, p. 39). ${ }^{37}$ Cf. APW I 1, document $n^{\circ}$ 5, ici p. 108-111; c'est le texte terminé sous la direction de Mazarin, avec les divergences par rapport aux projets de Richelieu signalées en bas de page. 
comme les affaires les plus importantes traitées dans cette section ${ }^{38}$. Parmi les états de l'Empire, la section XV évoquait nommément la landgravine de HesseCassel et les ducs de Brunswick et Lunebourg dont les plénipotentiaires de France devaient prendre »un soing particulier « ${ }^{39}$, sans qu'elle se prononçât de manière plus précise sur ce sujet. Pourtant, la Hesse-Cassel faisait partie des principaux alliés allemands de la France, et les ducs Frédéric de Lunebourg et Auguste de Brunswick-Wolfenbüttel passaient toujours pour alliés aux couronnes de France et de Suède malgré les traités de neutralité qu'ils avaient été obligés de conclure avec l'empereur en 1642. D'une façon générale, on constate que les instructions étaient très floues à l'égard de l'Allemagne, et cette imprécision ne s'explique certainement pas uniquement par le désir de garder une certaine flexibilité à l'avenir, mais elle apparaît bien comme une capitulation devant le nombre important et la complexité des affaires allemandes; au lieu de donner des recommandations précises aux ambassadeurs français, le gouvernement se limitait même à leur ordonner de se renseigner sur place, en consultant les envoyés des alliés allemands de la France, comme il est écrit tout au début de la section XV:

Quant aux intérestz de l'Allemagne [disait en effet le texte de 1643 (qui correspondait presque littéralement au projet de Richelieu terminé en février ou en mars 1637!)] il sera difficile d'en donner de bonnes instructions, à cause de la multitude et diversité d'iceux, et en effect $M^{r s}$ les Ambassadeurs les apprendront mieux sur les lieux de ceux qui seront envoyéz de tous les Princes qu'on ne pourroit les leur faire sçavoir par la présente instruction ${ }^{40}$.

Or, tout en ne montrant pas véritablement un désir de trop s'immiscer dans les affaires intérieures du Saint Empire romain germanique, auxquelles Richelieu et Mazarin ne semblaient pas s'intéresser si le pouvoir impérial et les compétences juridiques des ordres n'étaient pas concernés, cette section précisait encore une fois que c'était une conditio sine qua non pour la signature de la paix que la France ne fût pas obligée de renoncer aux alliances conclues avec les princes d'Allemagne et avec la couronne de Suède, puisque cette obligation rendrait la paix caduque, comme Richelieu l'écrivait en 1641 et $1642^{41}$. À l'appui d'une documentation que Richelieu avait collectionnée depuis les années 1630, le cardinal pouvait déduire le caractère constitutionnel du droit d'alliance des princes de l'Empire, qui, selon lui, était une prérogative que les liens de féodalité soumettant

38 Toutefois, Mazarin ne reprit pas l'argumentation de Richelieu selon laquelle il importait non seulement à la France, mais à la chrétienté en général que la Suède obtînt des territoires en Allemagne du Nord afin qu'elle pût à l'avenir limiter la puissance de la maison d'Autriche, ainsi que l'avait vu Richelieu en 1641 et 1642 (ses projets antérieurs n'avaient pas compris ce paragraphe), cf. ibid., p. 108.

39 Ibid.

40 Ibid., pour la version de 1637 , cf. ibid., document no 3 , ici p. 47 : la seule différence est qu'en 1637, Richelieu avait parlé d'une "grande diversité» des intérêts allemands tandis qu'en 1643, Mazarin parlait seulement de leur »diversité«; le mot "grande« ne fut retranché qu'après la mort de Richelieu.

${ }^{41}$ Ce passage a été conservé littéralement par Mazarin en 1643 à une seule différence près (qui est d'ailleurs purement stylistique); quelques nuances substantielles de l'ébauche originale par rapport à la version définitive datent encore du ministère de Richelieu. 
les états à l'empereur en tant que suzerain ne remettaient pas en cause. Le paragraphe minuté au moins partiellement manu propria par Richelieu fut littéralement conservé par Mazarin ${ }^{42}$. En réalité, ce droit d'alliance était bien revendiqué par certains états de l'Empire, mais il était loin d'être unanimement accepté et, en particulier, n'était pas respecté par l'empereur.

Les deux sections suivantes concernent les cas particuliers de trois princes de l'Empire: Palatinat, Bavière, Wurtemberg. Le différend existant depuis plus de deux décennies entre le prince palatin du Rhin, anciennement électeur du Saint Empire romain germanique avant que Frédéric $\mathrm{V}$ ne fût dépouillé de sa dignité au profit du duc de Bavière, et ce dernier, constituait une affaire délicate pour la France, comme nous l'avons déjà souligné, puisque Richelieu et Mazarin ne pouvaient pas abandonner la cause du palatin, mais voulaient gagner l'amitié de Maximilien de Bavière. Sans se prononcer sur le caractère bien ou mal fondé du transfert de la dignité électorale palatine, les instructions françaises (en l'occurrence la section XVI) déclaraient qu'il était impossible de donner des ordres précis. Elles se limitaient à enjoindre aux ambassadeurs français de ne pas s'opposer ouvertement à la restitution intégrale ou partielle du palatin, jugée inévitable, mais de soutenir discrètement les intérêts de la Bavière dans la mesure où elle favoriserait la résolution des questions concernant la France. En outre, elles leur demandaient surtout la plus grande discrétion afin de ne pas porter atteinte à la réputation du roi de France. Une autre difficulté consistait en la réciprocité des concessions: on pouvait craindre que si le duc de Bavière restituait le Haut-Palatinat au prince palatin, les adversaires de la France ne lui demandent de restituer la Lorraine à son ancien seigneur; mais comme Richelieu et Mazarin estimaient qu'ils avaient des preuves suffisantes pour démontrer la légitimité des prétentions françaises sur la Lorraine, ils permettaient aux ambassadeurs de France de consentir ouvertement à la restitution du Haut-Palatinat si le duc de Bavière en faisait l'of$\mathrm{fre}^{43}$. En ce qui concerne le cas du Wurtemberg, évoqué dans la section XVII, les ordres donnés aux diplomates ne sont pas plus précis qu'à l'égard du Palatinat et de la Bavière. Au contraire, on demande aux ambassadeurs de prendre leurs décisions sur place. Comme l'Autriche avait occupé une partie des terres du duc de Wurtemberg au cours de la guerre, voire les avait données à titre de gratifications à des généraux de l'armée et à des ministres impériaux, les plénipotentiaires français pouvaient s'opposer plus ou moins fermement aux revendications autrichiennes en fonction des concessions de l'empereur sur d'autres points ${ }^{44}$. Les cas très nombreux des autres états de l'Empire, qui demandaient la restitution des territoires et des droits qu'ils avaient perdus pendant la guerre (il suffit de lire l'article IV du traité d'Osnabrück pour se rendre compte de leur nombre considérable; traité qui ne mentionne pas les demandes refusées), ne sont évoqués que très sommairement, dans la section $X_{\text {VIII }}^{45}$.

42 APW I 1, document no 5, ici p. 109.

43 Cf. ibid., p. 109-110.

$44 \mathrm{Cf}$. ibid., p. 110 la section XVII, qui ne compte pas plus de quatre lignes et demie.

${ }^{45}$ En effet, la section XVIII, qui est encore plus courte que la section précédente, ne donne 
Dès lors, l'imprécision des informations et des ordres donnés aux diplomates apparaît de manière flagrante. En revanche, il faut souligner qu'en aucun cas ces instructions ne donnent des renseignements erronés sur la situation existant en $\mathrm{Al}-$ lemagne; la seule assertion qui n'est pas tout à fait correcte concerne la prétendue garantie constitutionnelle du droit d'alliance des états de l'Empire: au lieu d'un droit avéré, il s'agissait en réalité d'une prétention des ordres. Le fait que les projets d'instructions minutés par Richelieu et le texte final du 30 septembre 1643, rédigé sous la direction de Mazarin, ne se prononcent pas précisément sur une très grande partie des affaires intérieures de l'Allemagne ne permet pas de dire qu'il s'agisse d'un manque de connaissances. En fait, on peut en conclure que ni Richelieu ni Mazarin ne voulaient donner une nouvelle Constitution au Saint Empire romain germanique ou modifier profondément ses structures politiques, voire remettre en question son existence même - comme une partie de l'historiographie allemande l'a prétendu avant 1945. Il est évident que les deux cardinaux n'eurent que certains objectifs très précis: restreindre, dans une certaine mesure, l'autorité de l'empereur, sans l'abaisser trop fortement, faire interdire l'élection de son successeur de son vivant, assurer aux états de l'Empire certains droits politiques, notamment leur droit d'alliance, et les associer à l'empereur, sur le plan du droit international, afin qu'ils représentent ensemble le Saint-Empire quand il s'agissait de décider de la guerre ou de la paix. On ne trouve trace, avant 1648 , d'aucun programme de politique allemande allant au-delà de ces points très précis: ni dans les instructions de 1643, ni dans les projets préalables rédigés par Richelieu, ni dans aucun autre document diplomatique français. Le texte du 30 septembre 1643 n'évoque aucunement les autres droits politiques des états de l'Empire ni les prétentions des protestants concernant le droit de religion; certes, d'autres documents y sont consacrés, mais sans en faire une revendication centrale de la diplomatie française dans les négociations avec les Impériaux.

Nous constatons que Mazarin ne défendait pas toujours avec la même vigueur que son prédécesseur les projets d'une ligue allemande, comme le montre surtout un passage retranché du texte de Richelieu, mais qu'il ne donna pas d'ordres plus précis aux plénipotentiaires français en ce qui concerne les intérêts que la France pouvait avoir en Allemagne. À l'égard du Saint-Empire, il n'ajouta aucun passage substantiel aux projets que Richelieu lui avait laissés. Hermann Weber conclut à juste titre que cette sinstruction du 30 septembre 1643, quoique rédigée après la mort du cardinal [de Richelieu], représente dans son ensemble non seulement les idées de Richelieu, mais pour une bonne part le texte même tel qu'il avait été conçu par lui« 46 . On peut même aller plus loin et constater - comme le fait Weber - une continuité des buts de la politique allemande de Richelieu définis dans ses »avis« de 1625 et de 1629 jusqu'à sa mort, voire jusqu'à 1643 à travers les projets d'instructions qu'il avait légués à Mazarin. À l'image de Pignerol, qui permettait

aux diplomates que quelques renseignements concernant la conduite à tenir en général, en leur demandant d'en prendre »cognoissance sur les lieux« (ibid., p. 111).

46 WEBER, Richelieu et le Rhin, p. 267. En effet, cette conclusion est fondée sur la comparaison entre l'instruction terminée sous Mazarin et les avant-projets du ministériat de Richelieu. 
aux troupes françaises d'entrer en Italie, Richelieu voulait acquérir une entrée en Allemagne: ce fut d'abord Strasbourg, puis Brisach, forteresse protégeant le passage du Rhin. En général, il s'agissait d'acquérir des étapes et des passages dans la zone stratégique s'étendant de la Lorraine jusqu'au Rhin; l'intérêt du cardinal n’était en effet »pas orienté vers le Rhin, mais vers ses passages«. Il ne le convoitait pas »pour en faire la frontière du royaume de France «, comme ce fut le cas des projets d'>arrondissement ` du royaume conçus dans la deuxième moitié du XVII siècle, mais la demande de Brisach et des étapes était dictée par la nécessité d'avoir des passages permettant une intervention sûre et rapide en Allemagne ${ }^{47}$. Bien entendu, la forteresse de Brisach était censée servir d'entrée en Allemagne, non seulement à des fins défensives (protection des frontières du royaume), mais en vue d'une éventuelle intervention militaire. D'ailleurs, cette éventualité attribuerait à la France - sans qu'elle soit forcément réalisée - une influence et un rôle politiques dans l'Empire. Cela veut dire qu'après la conclusion de la paix, Richelieu voulait garder la mainmise de la France sur les affaires intérieures de l'Allemagne, et il est certain que son objectif était le soutien des princes et la défense de leur liberté contre la puissance des Habsbourg. En effet, cette intention est très clairement formulée dans l'instruction du 30 septembre 1643: »on pourra doucement faire concevoir à ceux, avec lesquelz on traittera, qu'ainsy que Pignerol est nécessaire au Roy comme une porte pour le secours de l'Italie, ainsy il est important que Sa Majesté ne soit pas séparée de l'Allemagne, affin qu'elle soit en estat de ne souffrir pas les oppressions qu'on pourroit faire à divers Princes qui y possèdent des Estatz « ${ }^{48}$. En conformité avec ces principes, après la paix, la France ne se désintéresserait donc pas de l'Allemagne. Tout en étant placée sous le signe de l'antagonisme avec l'Espagne, la politique de Richelieu jeta ainsi les fondements d'une politique française durable dans le Saint-Empire, politique qui devait tenir compte des évolutions constitutionnelles et y prendre parfois un rôle actif afin de soutenir et de protéger les ordres.

Si l'argumentation de l'instruction du 30 septembre 1643 était censée fournir des raisons aux plénipotentiaires français pour justifier les demandes territoriales de la France envers les princes allemands (il s'agissait donc »d'arguments adressés aux princes allemands afin de les faire consentir à ce que la France obtienne ce qu'elle désire $\aleph^{49}$ ), on peut exclure de se trouver en face d'un camouflage pur et simple des prétendues > vraies intentions « du cardinal, puisque, dès 1625 et 1629 , il avait formulé des idées similaires dans des documents internes qui s'adressaient au Conseil du roi et à Louis XIII en personne. Considérant, au moins depuis 1625 , que la France avait tout intérêt à suivre de près les affaires d'Allemagne »tant pour la protection de ses alliés que pour empêcher que ces pais ne tombent absolument soubz l'entière domination de la Maison d'Autriche«, il exprima déjà en 1629 l'idée selon laquelle elle devait impérativement »bâtir et s'ouvrir des portes

47 Ibid., p. 269.

48 APW I 1, no 5, "Section première«, p. 63.

49 WEBER, Richelieu et le Rhin, p. 270. 
pour entrer dans tous les États de ses voisins et les pouvoir garantir de l'oppression d'Espagne «50. La logique de l'instruction du 30 septembre 1643 obéit à cette disposition impérative.

De 1637 à décembre 1641, les projets d'instructions pour les ambassadeurs français au congrès général de la paix contiennent - littéralement ou en substance - le passage suivant: "Estant impossible de diviser la Maison d'Autriche, séparant celle d'Alemagne des intérests de celle qui est en Espagne, il est impossible de trouver une seureté en la Paix qui soit physique, et partant il faut se contenter de celle qui se peut trouver dans une ligue «51. Le fait que cette prédiction se soit révélée incorrecte explique que la diplomatie française n'ait pas accordé la même importance aux projets de ligues en Westphalie que ne l'avait fait le cardinal de Richelieu. La garantie qui fut effectivement stipulée par les traités de Westphalie constitue bel et bien »une garantie collective des signataires «, mais elle n'est pas comparable au système de sécurité collective imaginé par Richelieu, système qui reposait sur des ligues et supposait la conclusion d'une paix générale - ce que la paix de Westphalie ne fut pas ${ }^{52}$. À l'égard de l'élément le plus essentiel de la conception de la paix chrétienne, il n'y a donc pas de continuité parfaite allant de Richelieu aux traités de Münster et d'Osnabrück.

Richelieu avait d'ailleurs sous-estimé les difficultés que les négociations sur les ligues italienne et allemande susciteraient. À l'égard de la première, les projets rédigés sous son ministère avaient précisé que le grand-duc de Toscane l'avait luimême demandée quelques années auparavant. En ce qui concerne la ligue allemande, le cardinal avait supposé que les princes d'Allemagne auraient un très grand intérêt à la conclure:

Les Princes de l'Empire la doivent désirer, puis que par ce moyen leurs privilèges leur seront conservéz et plus considéréz, et qu'il ne sera pas permis à ''Empereur toutes fois et quantes qu'il luy plaira de mettre de grandes armées sur pied, ou pour les opprimer, ou pour se porter à des guerres qui n'ont autre but que les avantages de l'Espagne ${ }^{53}$.

Comme nous le savons, cette estimation se révéla erronée, et c'est peut-être pour cela que Mazarin, devenu plus sceptique à cet égard que ne l'avait été son prédécesseur, n'inséra pas ce paragraphe dans l'instruction de 1643. Une certaine discontinuité se manifeste aussi dans le fait qu'en novembre 1645 Mazarin envoya

50 Passages des avis de 1625 et de 1629 , cités ibid.

51 Cf. APW I 1, no 5, $\gg$ Section quatriesme «, p. 71. Weber se trompe en disant que ce passage fut encore noté en marge des instructions du 30 septembre 1643, cf. WEBER, Richelieu und das Reich, p. 45. Selon les éditeurs du document, ce passage, encore noté en marge du projet B3 (décembre 1641), ne le fut plus sur le projet C1 (fin de l'été 1643).

52 Pour ces raisons, nous ne pouvons pas approuver la remarque suivante de Hermann Weber: „Ce système d'une paix générale, garantie par les partenaires, sera r[é]alisé après la mort de Richelieu dans les Traités de Westphalie, mais sur la base des instructions, que Richelieu avait déjà rédigées, et qui depuis avaient été reprises par Mazarin«, cf. WEBER, Dieu, le roi et la chrétienté, p. 241.

$53 \mathrm{Cf}$. le dernier projet du temps de Richelieu mis au net par Mazarin avant que celui-ci ne prenne la direction des affaires du royaume, dans APW I 1, $\mathrm{n}^{\circ} 5$, ici p. 71. 
aux ambassadeurs français une instruction supplémentaire modifiant en quelque façon les instructions qui leur avaient été données en $1643^{54}$.

La version de l'instruction française que Richelieu avait rédigée en février et mars $1637^{55}$ ne s'expliquait pas plus précisément sur les affaires d'Allemagne que l'instruction de 1643. Le projet de 1637 nommait les intérêts des princes d'Allemagne et des Suédois (c'est-à-dire les intérêts des Suédois en Allemagne) en dernier lieu parmi les objets de la négociation. Elle définissait comme but principal de la politique française au congrès de la paix la formation d'une ligue des princes catholiques et protestants de l'Empire pour que la maison d'Autriche ne puisse pas établir son autorité absolue sur l'Allemagne et que l'armée de l'Empire ne soit pas instrumentalisée par l'Espagne pour combattre ses adversaires, et notamment la France. Richelieu supposait qu'il n'était pas difficile de convaincre les princes allemands (même les adhérents des Habsbourg dans l'Empire) de l'utilité de cette ligue, puisqu'elle devait assurer leur liberté et leur sécurité ainsi que le respect général du traité de paix ${ }^{56}$. Or, cet espoir de Richelieu était mal fondé: les princes de l'Empire ne désiraient pas une telle ligue et, par conséquent, n'ont pas soutenu cette demande en Westphalie.

Richelieu voulait apporter le soutien de la France aux Suédois dans les négociations sur leur dédommagement territorial, mais il ne désirait leur présence en Allemagne qu'à moyen terme, puisqu'il jugeait que les ligues d'Allemagne et d'Italie devaient prendre le relais de la puissance suédoise en maintenant la paix dans l'Empire et en Europe. Le texte ne s'expliquait pas sur la position de la France vis-à-vis de la présence des Suédois à long terme. On peut supposer que Richelieu ne la souhaitait que dans la mesure où elle était nécessaire pour contrebalancer la puissance des Habsbourg. Il prévoyait que non seulement l'empereur, mais aussi les princes d'Allemagne s'opposeraient au principe de dédommagement de la Suède en territoires allemands et préféreraient lui donner de l'argent; or, un tel dédommagement pécuniaire ne servait pas les intérêts français, parce qu'après le départ des Suédois, les Habsbourg et en particulier les Espagnols risquaient de reprendre le pouvoir en Allemagne au préjudice de la sécurité du royaume de France si la paix n'avait, auparavant, pas encore été solidement ancrée en Europe et en Allemagne par l'instauration et le bon fonctionnement des deux ligues. Cependant, il était évident que ces négociations seraient très difficiles

54 Ce document est publié en annexe dans: Franz BosBach (éd.), Acta Pacis Westphalicae, série II, section B: Die französischen Korrespondenzen, vol. 2: 1645, avec le concours de Kriemhild Goronzy et de Rita BoHLEN, Münster 1986 (APW II B 2), document $n^{\circ} 267$, p. 872-890.

55 Cf. APW I 1, document $n^{\circ} 3$, p. 38-55. Comme en 1643, les questions allemandes n'étaient pas évoquées au début de l'instruction. Les différents thèmes y étaient traités selon l'ordre suivant: les problèmes de préséance et les modes de négociation; les conquêtes en Espagne, en Italie, aux Grisons; la Lorraine et les Trois-Évêchés; les Pays-Bas; les affaires d'Allemagne (dont le dédommagement de la Suède); la sécurité collective; la tactique de la négociation; les documents que les députés français étaient censés emporter en Allemagne. 56 Cf. ibid., p. $40,47$. 
du fait de la convergence des intérêts allemands et espagnols ${ }^{57}$. Le dédommagement territorial de la Suède était donc un élément indispensable pour la sûreté du traité de paix. La politique française qui soutenait cette demande suédoise était fondée sur la supposition qu'après sa conclusion les Habsbourg tenteraient de nouveau d'établir leur autorité souveraine sur l'ensemble des états de l'Empire au profit des intérêts espagnols. Pour Richelieu, le Saint-Empire restait donc avant tout l'arrière-cour de l'Espagne.

En ce qui concerne les principaux différends qui devaient être réglés dans l'Empire, l'instruction ne se prononçait pas sur la restitution du prince palatin, mais elle jugeait sa dépossession illégitime. Toutefois, Richelieu considérait que la France devait en tout cas soutenir la Bavière de manière ferme, mais discrète ${ }^{58}$. De toute évidence, ce point était délicat pour la France; mais, tout compte fait, il importait au roi de pouvoir compter un puissant prince catholique parmi ses alliés dans l'Empire, tout en ne choquant pas ses alliés protestants. Cette instruction établissait le principe de la réciprocité des restitutions qui pouvaient être demandées concernant la Lorraine d'un côté, le Palatinat et le Wurtemberg de l'autre ${ }^{59}$. À cet égard, on note donc une différence importante entre les versions de 1637 et de 1643, puisqu'en 1637, Richelieu conseillait aux ambassadeurs français de demander la restitution du Palatinat, du Wurtemberg et d'autres conquêtes habsbourgeoises si la maison d'Autriche demandait celle de la Lorraine, tout en contestant le fondement juridique de cette revendication. En revanche, en 1643, on évitait soigneusement d'établir un parallèle entre ces différents cas, puisque cette argumentation risquait de tourner au préjudice de la France, qui désirait que ses alliés allemands obtiennent la restitution de leurs biens, mais voulait conserver la Lorraine. En 1643, on insistait donc sur les différences qui s'opposaient à un règlement identique des problèmes lorrain et palatin, tandis qu'en 1637 Richelieu avait encore proposé de régler la position française concernant le Haut-Palatinat et le Wurtemberg en fonction des concessions des Habsbourg sur la Lorraine ${ }^{60}$.

Tout en évoquant Philippe-Christophe de Sötern comme dernier prince allemand pour lequel la France devait demander la restitution, Richelieu attachait évidemment la plus grande importance à ce que ce prince obtînt satisfaction. Étant donné que le cas du prince électeur de Trèves avait servi de légitimation à l'intervention française dans l'Empire deux ans auparavant, en 1635, la France risquait sa crédibilité auprès des princes d'Allemagne si la protection qu'elle avait accordée à Sötern s'avérait inefficace, de sorte qu'en 1643 l'argumentation de l'instruction française ressemblait fortement à l'article rédigé en 163761 . C'était un point sur lequel la France devait tenir ferme, abstraction faite de toute conjoncture politique à court terme. C'est pourquoi la version définitive de cet article était encore

57 Cf. ibid., p. 47.

58 Cf. ibid., p. 48.

59 Cf. ibid., p. 45.

${ }^{60} \mathrm{Cf}$. ibid., p. 48, au sujet du problème palatin, cf. aussi ibid., l'article relatif au Wurtemberg, analogue à la Bavière.

${ }^{61}$ Cf. ibid. 
plus précise sur certains aspects que le projet de 1637. Mais, en principe, elle reposait sur la même argumentation: l'empereur n'ayant pu protéger l'électeur de Trèves et la religion catholique dans son évêché, le roi avait légitimement pris leur défense, puisque l'électeur lui avait demandé sa protection. Juridiquement, cette conception se fondait donc sur l'idée du caractère subsidiaire de la royauté française par rapport à l'office de l'empereur.

En ce qui concerne l'Italie, où Richelieu attendait un pareil engouement pour ses projets de ligues, la France ne reconnaissait pas la suzeraineté de l'empereur et de l'Empire sur Pignerol, jugeant que leur consentement à la cession de cette place n'était pas indispensable ${ }^{62}$.

Outre le projet de la ligue des princes d'Allemagne et les intérêts particuliers des princes mentionnés, l'instruction de 1637 abordait le problème de l'élection impériale. Or, contrairement à la demande d'interdiction de l'élection vivente Imperatore de la part de la diplomatie française en Westphalie, ce texte fixait seulement la ligne de conduite que les ambassadeurs français devaient adopter pour se prononcer sur l'élection de Ferdinand III. Le roi de France contestait sa validité, mais le texte de l'instruction était peu précis sur ce point et demandait aux diplomates de concerter leur position avec les alliés suédois et allemands de la France. Ils étaient autorisés à promettre que la France ne s'opposerait pas à l'élection de Ferdinand III après la conclusion de la paix, à condition que les formalités prévues par la Constitution soient respectées ${ }^{63}$. Le problème constitutionnel de l'élection du roi des Romains du vivant de son prédécesseur n'y était pas évoqué. Cela confirme l'absence d'un véritable programme de la diplomatie française pour changer les données constitutionnelles dans le Saint-Empire. Richelieu envisageait des cas concrets en rédigeant les projets de l'instruction pour le congrès de la paix prévu à Cologne.

D'ailleurs, l'instruction de 1637 contenait un catalogue des documents et des ouvrages que les ambassadeurs français étaient censés emporter en partant pour Cologne. Â l'égard de l'Allemagne, cette liste ne mentionnait aucune pièce relative à la Constitution du Saint-Empire en général. On demandait aux ambassadeurs de se munir de documents concernant les libertés des électeurs, le cas de

62 Cf. ibid., le paragraphe concernant Pignerol, p. 41-42, en particulier p. 42.

63 Cf. ibid., p. 49. De toute évidence, cette remarque sur les formes de l'élection répétait ce que les Français avaient reproché à Ferdinand III au lendemain de la diète électorale, à savoir que toutes les formalités fixées par les constitutions de l'Empire, notamment dans la Bulle d'or, n'avaient pas été respectées lors de son élection. D'ailleurs, la première version de l'instruction avait été terminée avant cette élection; elle l'avait donc seulement envisagée comme une possibilité, non comme un fait accompli. Richelieu avait enjoint aux diplomates français de ne pas s'y opposer à condition que les électeurs y soient favorables et qu'elle soit remise au lendemain de la conclusion de la paix. Au cas où les électeurs y seraient peu favorables, le cardinal leur avait demandé de »fomenter« l'opposition de ces derniers, mais de manière discrète (»sans s'en rendre ouvertement partisans «). Toutefois, Louis XIII leur avait confié qu'il ne voulait pas empêcher cette élection après la conclusion de la paix et, en particulier, des deux ligues respectivement formées par les princes d'Allemagne et d'Italie (ibid., le texte de la première version est cité en bas de page). 
l'électeur de Trèves en particulier, le Palatinat et le Wurtemberg, ainsi que l'élection de Ferdinand III ${ }^{64}$.

Pendant tout le temps de son ministère, le caur de la politique allemande de Richelieu reposait, du moins depuis l'entrée de la France en guerre, sur le système de la sûreté collective fondé sur les ligues allemande et italienne. En 1636 et 1637, le cardinal était très optimiste à l'égard des chances de faire réussir ce projet. Il supposait que les princes d'Allemagne y consentiraient pour plusieurs raisons: cette ligue leur assurerait le respect de leurs privilèges. L'empereur ne pourrait plus imposer ses décisions aux états de l'Empire, au préjudice de leur autorité; il n'aurait plus, en particulier, le droit de lever des armées sans le consentement des états (au moins des électeurs). De cette façon, l'Allemagne ne serait plus obligée de suivre la politique belliqueuse de l'Espagne, qui lui avait été imposée indirectement, par l'intermédiaire de l'empereur. Enfin, Richelieu estimait que Louis XIII pouvait même gagner l'affection des princes d'Allemagne en proposant cette ligue qui contribuait à garantir la paix ${ }^{65}$.

Nous avons déjà souligné le caractère erroné de cette estimation. Il convient désormais de mettre en relief un autre détail qui prouve que les objectifs de Richelieu en matière de droit constitutionnel allemand étaient incontestablement beaucoup plus limités qu'on ne l'a supposé $a$ posteriori. En effet, nous avons vu que Richelieu se contentait du consentement des électeurs pour que l'empereur pût déclarer la guerre et lever des troupes au nom de l'Empire; il envisageait l'accord des autres états de l'Empire comme une éventualité, non comme une nécessité. Sur ce point, les traités de Westphalie vont donc plus loin que les revendications constitutionnelles du cardinal. Il est certain que le droit constitutionnel de la paix de Münster et d'Osnabrück ne fut pas imposé à l'Allemagne par Richelieu (pas plus que par Mazarin). Certes, Richelieu visait une pax gallica, une paix qui servirait surtout le roi de France. Mais il n'était pas complètement faux de déclarer, en 1636 et 1637, qu'il ne prétendait à aucun territoire ni à rien d'autre en Allemagne. Cette déclaration que les ambassadeurs français devaient faire aux princes catholiques et protestants représentés au congrès de la paix était effectivement véridique, dans la mesure où le cardinal n'envisageait ni une expansion territoriale de la France au détriment de l'Allemagne ni un remaniement fondamental de la Constitution du Saint-Empire. In nuce, le cardinal esquissait dans ce contexte le système d'un équilibre ou du moins d'une "balance « entre les Habsbourg d'un côté et la France et les princes allemands et italiens pacifiques de l'autre66. L'instruction de 1637 reflétait parfaitement les idées politiques du cardinal en privilégiant la politique des "portes « et des "passages « à l'expansion territoriale systématique. Bien entendu, Richelieu ne renonçait pas pour autant à l'acquisition de nouveaux territoires s'ils revêtaient une importance stratégique majeure ${ }^{67}$.

64 Cf. ibid., p. 54. À ce sujet, l'instruction promettait aux ambassadeurs des mémoires particuliers rédigés par Stella de Morimont, secrétaire du comte d'Avaux.

65 Cf. ibid., p. 50.

$66 \mathrm{Cf}$. ibid.

67 En l'occurrence, Louis XIII revendiquait la cession de Pignerol et de la Lorraine. Cf. 
L'agrandissement territorial devait contribuer à la sûreté du royaume et de ses alliés. Or, à cet égard, les demandes françaises évoluèrent jusqu'à l'ouverture du congrès de Westphalie.

En ce qui concerne les objectifs de la politique allemande au moment de la rédaction des premiers projets d'instructions, on peut constater qu'une note écrite probablement en 1638 (très certainement avant l'été 1641), dans laquelle étaient précisées certaines conceptions françaises de la paix, ordonnait aux ambassadeurs de France de soutenir les demandes des princes, états et villes alliés de la France, et de ceux qui se déclareraient neutres ou auraient recours à la France en abandonnant le parti de l'empereur ${ }^{68}$. En revanche, ce texte ne fixait aucun objectif précis en matière de politique constitutionnelle allemande. Cette note confirme notre observation relative à l'instruction de 1637, dans laquelle le roi de France prétendait pouvoir remplacer l'empereur en tant que protecteur des princes de l'Empire. En effet, selon cette note, le parlement de Metz était sur un pied d'égalité avec le Conseil aulique de Vienne. On reprochait à l'empereur d'avoir empiété sur les droits et sur la compétence judiciaire des états de l'Empire en évoquant au Conseil aulique des cas qui auraient dû être décidés par la Chambre impériale de Spire ${ }^{69}$. Cette comparaison entre le parlement de Metz et le Conseil aulique était injustifiée dans la mesure où ce dernier était l'un des deux tribunaux souverains de l'Empire et qu'il y avait une certaine concurrence entre celui-ci et la Chambre impériale de Spire; dans la plupart des cas, les compétences des deux cours étaient identiques. Mais le rédacteur de la note se souciait probablement moins de la pertinence de la comparaison que de sa fonction, c'est-à-dire la légitimation de l'existence du parlement de Metz et du fait qu'on y évoquait des causes relevant de la compétence de la Chambre impériale.

De toute évidence, les Français tentaient d'imposer leurs institutions (roi, parlements) comme subsidiaires ou juridiquement égales aux institutions de Vienne (empereur, Conseil aulique). D'un côté, il semble bien qu'ils aient ainsi cherché à ouvrir le Saint-Empire à l'influence française; de l'autre, il est évident qu'ils ont voulu faire apparaître l'empereur comme un étranger ne faisant pas véritablement partie du corps germanique. Les »droitz de l'Empire« que cette note mentionne sont les droits des états de l'Empire; ce sont eux qui représentent l'Empire. L'em-

ibid.: «On pourra doucement faire concevoir à ceux, avec lesquelz on traittera, qu'ainsy que Pignerol est nécessaire au Roy comme une porte pour le secours de l'Italie, ainsy Sa Majesté désire plus n'estre séparée de l'Allemagne par la Lorraine pour cette raison que pour aucun désir qu'elle ait de s'agrandir«.

68 Cf. ibid., document n ${ }^{\circ} 4$, p. 56-58, ici p. 57, le paragraphe intitulé »Sur l' article qui traitte les intérestz de l'Allemagne«.

69 Cf. ibid., p. 57, le paragraphe »Pour le Parlement de Metz: Encores qu'il soit à Thoul, il ne laisse pas de s'attribuer les appellations qui alloient à Spir lors de son establissement«. La note proposait la réplique suivante à cette assertion: »L'on peut dire au contraire que la Maison d'Austriche a énervé la mesme Chambre de Spir en attribuant au Conseil Aulique de l'Empereur les principaux droitz de l'Empire qui relevoient de Spir, ce que le Roy désire estre restably en faveur de la liberté germanique«. 
pereur est tout à fait marginalisé. Le Conseil aulique de l'Empire devient, dans cette rhétorique, le »Conseil Aulique de l'Empereur«.

Dans cet article, Louis XIII précise un des objectifs de la France concernant la politique intérieure allemande: le rétablissement du bon fonctionnement de la Chambre impériale de Spire, qui est présenté comme un moyen pour rétablir la liberté des états. Mais dans l'instruction de 1643, on ne recopia pas ce paragraphe.

Or, en étudiant les conceptions françaises de la paix, il convient d'analyser non seulement les buts de la politique de la France en matière de droit constitutionnel allemand, mais aussi les sources qui ont été utilisées ou qui ont été recommandées aux ambassadeurs censés représenter le roi au congrès de la paix. Nous avons pu noter qu'à la fin des premiers projets de l'instruction principale, on avait ordonné aux plénipotentiaires de se procurer certains documents justifiant les revendications de Louis XIII. Dans la version du projet d'instructions rédigée entre début août et mi-septembre 1641 , ce catalogue porte un titre particulier $^{70 ;}$ il ne s'agit donc plus d'une partie de l'instruction même, mais d'une annexe qui ne sera plus intégrée dans les versions suivantes. On peut supposer que ces renseignements pratiques ont été donnés aux ambassadeurs a parte. Quoi qu'il en soit, ce texte fut minuté par Cherré, secrétaire de Richelieu, et revu par son collègue Charpentier ainsi que par Richelieu en personne ${ }^{71}$. Au début, le »Roole des pièces« mentionne les documents servant à légitimer les demandes territoriales de la France en Italie et en Lorraine (en particulier un procès-verbal rédigé par Le Bret). En ce qui concerne les questions allemandes évoquées dans la deuxième partie (c'est-à-dire les électeurs, surtout l'archevêque de Trèves - point sur lequel la France devait tenir ferme, du moins officiellement $^{72}$ - le palatin, ainsi que le duc de Wurtemberg et l'invalidité de l'élection de Ferdinand III), les recommandations données aux ambassadeurs correspondent, en règle générale, à la version minutée au début de $1637 . \mathrm{Ni}$ en 1637 ni en 1641 Richelieu n'indiqua de documents ou d'ouvrages précis dans lesquels les plénipotentiaires français pouvaient puiser les informations concrètes. Il se limita à leur imposer un filtrage intentionnel: ils étaient obligés de choisir les documents en fonction des buts de la politique française.

$70 »$ Roole des pièces que Mess ${ }^{\text {rs }}$ les Ambassadeurs doivent nécessairement avoir avec eux «, publié ibid., document n ${ }^{\circ}$ 9, p. 136-139.

71 Selon les éditeurs du document, une grande partie des corrections fut écrite par le cardinal manu propria.

72 Toutefois, TISCHER note que la libération de l'électeur de Trèves et la restitution de ses biens et dignité n'avaient pas constitué le véritable mobile de l'intervention française, mais avait seulement servi à la légitimer officiellement. En vérité, cette revendication n'occupait pas une place centrale dans l'instruction française, EAD., Französische Diplomatie, p. 202. Pour les causes de la déclaration de guerre en 1635 , cf. les ouvrages indiqués dans le chapitre précédent ainsi que la mise au point sur ce problème publiée par David PARROTT, The Causes of the Franco-Spanish War of 1635-59, dans: Jeremy BLACK (dir.), The Origins of War in Early Modern Europe, Edinburgh 1987, p. 72-111. L'auteur juge que l'attaque de Trèves par les Espagnols et l'emprisonnement de Sötern ne constituèrent "qu'un incident parmi beaucoup d'autres « (»it was merely one incident among many«, ibid., p. 104). 
En ce qui concerne ces objectifs, on constate que Richelieu ne demandait pas que les Habsbourg fussent exclus de la dignité impériale et qu'il n'envisageait pas une expansion territoriale importante de la France au préjudice de la maison d'Autriche. De plus, il reconnaissait même la suzeraineté de l'Empire sur Metz, Toul et Verdun (à condition que l'Empire reconnût les droits de protection que la France y réclamait) et ne demandait pas la cession de l'Alsace ${ }^{73}$. En revanche, nous avons vu qu'il demandait l'admission des états de l'Empire au congrès de la paix, dans les négociations sur les préliminaires de Hambourg, menées sur place par le comte d'Avaux, avec la notable exception du duc de Lorraine. À la demande de la France, celui-ci n'y fut pas admis, car il était l'allié du parti habsbourgeois, tout en étant incontestablement un prince de l'Empire ${ }^{74}$. Cette admission des ordres et le soutien apporté à la protection de leurs droits étaient certainement les éléments essentiels de la politique allemande de Richelieu.

Malgré la mort de l'Homme Rouge avant l'ouverture du congrès de Münster, il y avait une continuité certaine de sa politique dans la diplomatie française au-delà de son décès. Tout d'abord, son successeur Mazarin avait été son proche collaborateur; on sait que Richelieu avait tenu des conférences avec ce dernier au sujet des préparatifs de ce congrès ${ }^{75}$. Par ailleurs, la France disposait en Allemagne d'un diplomate expérimenté, le comte d'Avaux, qui avait longtemps servi Richelieu, puis Mazarin, et qui, alors qu'il était plénipotentiaire français à Münster, montra à son collègue impérial Maximilien, comte de Trauttmansdorff, une instruction qu'il avait reçue du vivant de Richelieu au sujet de Brisach ${ }^{76}$.

Pour sa part, Mazarin conservait, dans l'instruction définitive de 1643, les arguments servant à l'auto-légitimation de la politique française que Richelieu avait donnés dans ses projets. Concernant l'expansion territoriale, ses arguments étaient de trois natures différentes: comme la France prétendait ne pas avoir commencé la guerre afin de faire des conquêtes durables, on justifiait ses prétentions territoriales surtout par ses anciens droits juridiques ou historiques sur plusieurs

73 Cf. Trscher, Französische Diplomatie, p. 196.

74 Un mémoire du roi, intitulé »Mémoire à Messieurs les Plénipotentiaires touchant l'affaire du duc Charles dans la négociation de la paix «, justifia ultérieurement le peu de considération que les Français avaient à l'égard du duc Charles, non par des arguments juridiques, mais par la faiblesse du duc et de ses protecteurs: mémoire de Louis XIV, Paris, 14 octobre 1646, Clivia Kelch-Rade, Anuschka Tischer (éd.), Acta Pacis Westphalicae, série II, section B: Die französischen Korrespondenzen, vol. 4: 1646, avec le concours de Kriemhild GoRONZY, Münster 1999 (APW II B 4), document no 198, p. 585-589, ici p. 587.

75 Mazarin l'avouait lui-même dans un mémoire écrit à Paris le 16 février 1646 et adressé à Longueville, d'Avaux et Servien, plénipotentiaires français en Westphalie de 1644/1645 à 1648/1649, cf. Elke Jarnut (†), Rita BoHLEn (éd.), Acta Pacis Westphalicae, série II, section B, vol. 3/1-3/2: 1645-1646, avec le concours de Kriemhild GORONZY et de Franz BOSBACH, Münster 1999 (APW II B 3), t. I, document no 115, p. 386-402, ici p. 394.

76 À propos d'une conférence qu'il avait tenue avec l'ambassadeur impérial, d'Avaux écrivait en effet à Mazarin de Münster, le 21 mai 1646: »ce que je luy [sc. Trauttmansdorff] dis pouvoir monstrer par escrit que dans l'instruction qui me fut donnée du vivant de feu monsieur le cardinal de Richelieu il y a ordre précis de ne faire jamais la paix sans garder Brisach«; ibid., document $n^{\circ} 282$, p. 984-991, ici p. 988. 
États de l'Europe; dans les cas où elle ne pouvait pas faire valoir d'anciens droits, on alléguait le droit de la guerre, le ius belli, qui justifiait au XVIIe siècle la rétention de territoires conquis par les armes; à l'égard de l'Italie et de l'Allemagne, on recourait à l'argument de la sûreté de la paix et de la protection ou du rétablissement des libertés des princes italiens et allemands pour légitimer certaines revendications territoriales françaises ${ }^{77}$. Cependant, Mazarin élargit notablement, en 1643, les demandes territoriales par rapport aux conceptions de Richelieu. En ce qui concerne l'Allemagne, on trouve pour la première fois dans l'instruction principale de 1643 la demande de retenir pour toujours, ou jusqu'à ce que l'empereur remplît d'autres conditions, les »places que Sa Majesté tient en Alsace, comme bien conquises par le droict de la guerre « 78 (le ius belli était certes la légitimation la plus problématique, mais elle était en général acceptée par les juristes du XVII' siècle).

Bien que les instructions écrites entre 1637 et 1643 soient des documents à usage interne, elles ne servaient pas uniquement à auto-légitimer les positions françaises ${ }^{79}$. Souvent, ces instructions étaient censées fournir des arguments que les ambassadeurs de France pouvaient faire valoir au congrès de la paix, de sorte que, très certainement, au moins une partie de ces justifications s'adressait non seulement aux hommes d'État et aux ambassadeurs français, mais aussi à leurs parties adverses et au public européen en général.

77 Cf. TisCHER, Französische Diplomatie, p. 202.

78 APW I 1, document $\mathrm{n}^{\circ} 5$, ici p. 75.

79 TISCHER insiste sur cet aspect de la justification interne, EAD., Französische Diplomatie, p. 202. 
